\title{
UMLS-based Comparison of Medical Forms
}

Results from: 07.02.2013

Number of Forms: 7

Number of Items: 285

Min. Number of Items per form: 5

Max. Number of Items per form: 87

Average Number of Items per form: 41

Domain: Prostate cancer 


\section{Identical Items}

QM Prostate Data Set

QM Cancer Diagnosis Data

HIS Surgery Urology

HIS Surgery Complications

HIS Pathological Report

HIS Medical History

Epidemological Registry
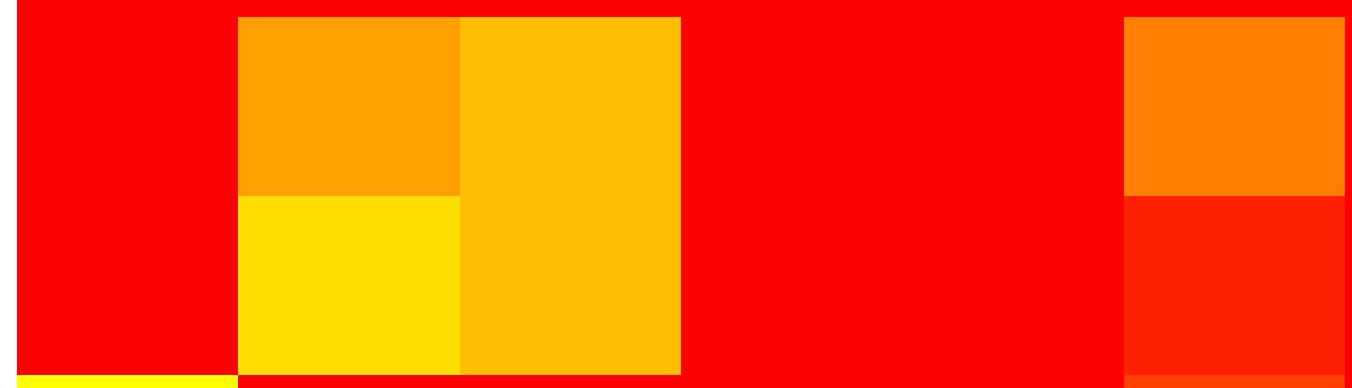

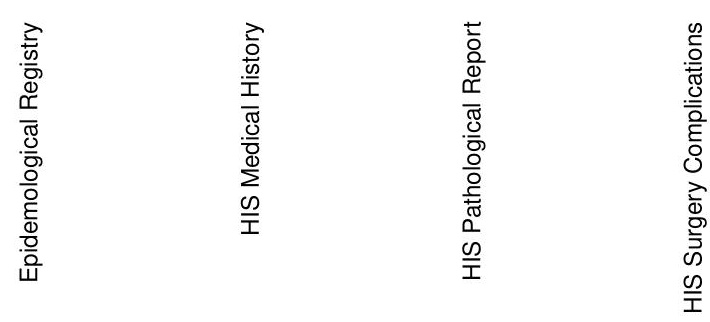




\section{Matching Items}

QM Prostate Data Set

QM Cancer Diagnosis Data

HIS Surgery Urology

HIS Surgery Complications

HIS Medical History

Epidemological Registry

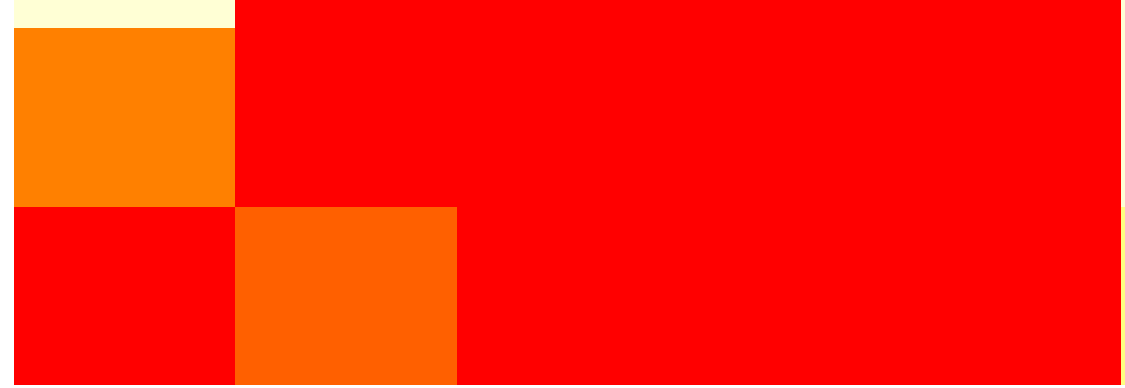

\begin{tabular}{|c|c|c|}
\hline 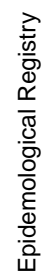 & 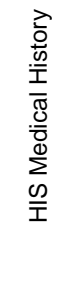 & 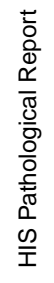 \\
\hline
\end{tabular}




\section{Similar Items}

QM Prostate Data Set

QM Cancer Diagnosis Data

HIS Surgery Urology

HIS Surgery Complications

HIS Pathological Report

HIS Medical History

Epidemological Registry
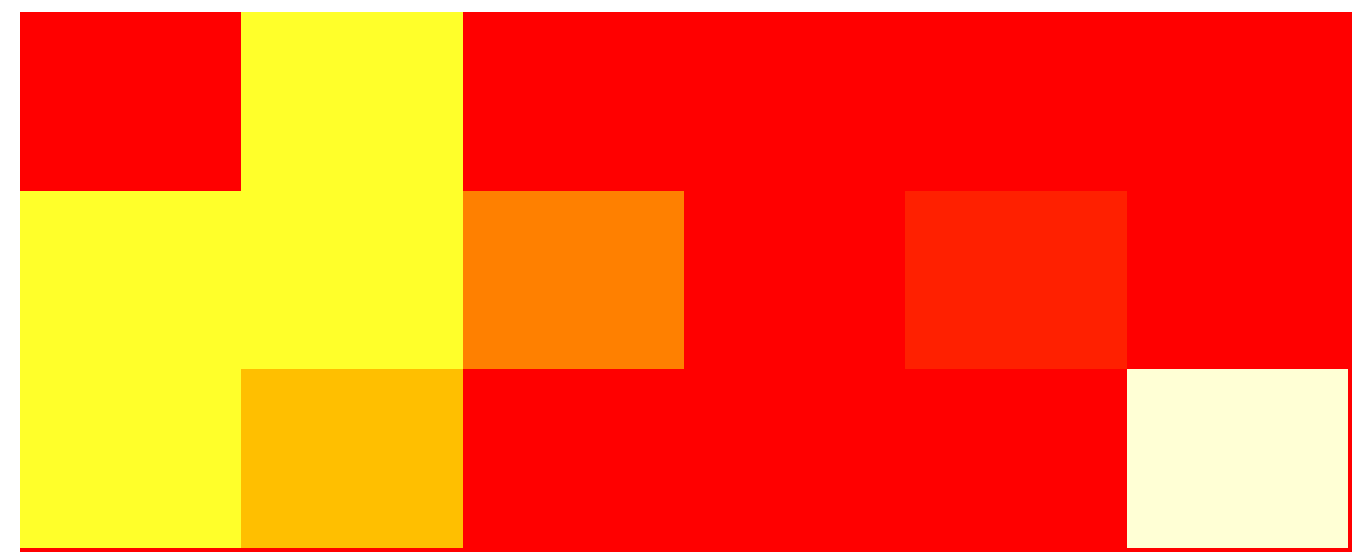

.
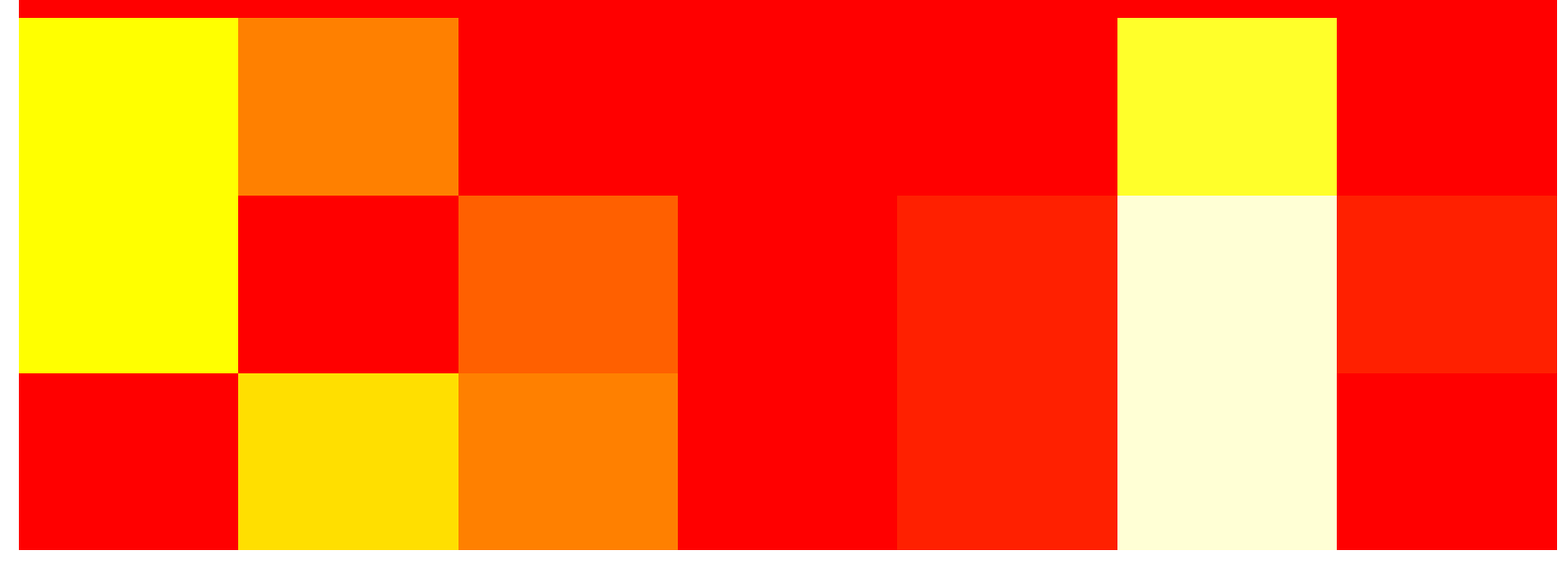

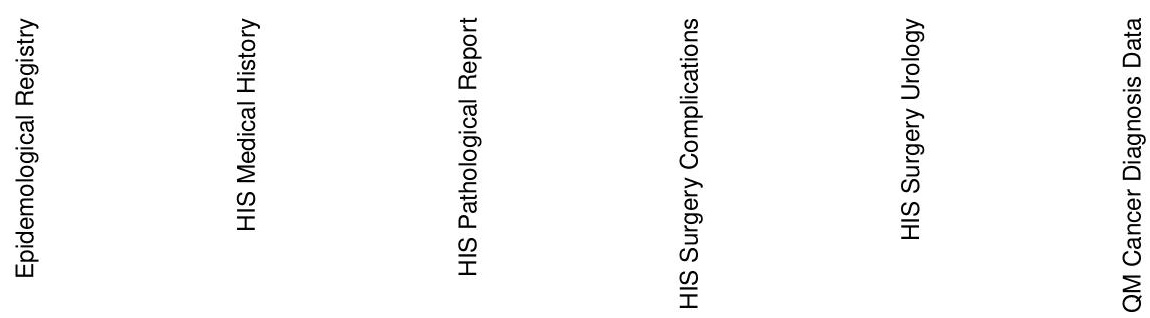


QM Prostate Data Set

QM Cancer Diagnosis Data

HIS Surgery Urology

HIS Surgery Complications

HIS Pathological Report

HIS Medical History

Epidemological Registry

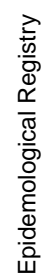

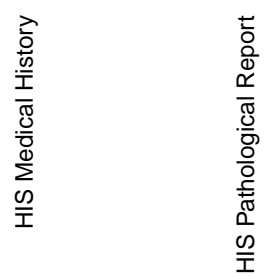




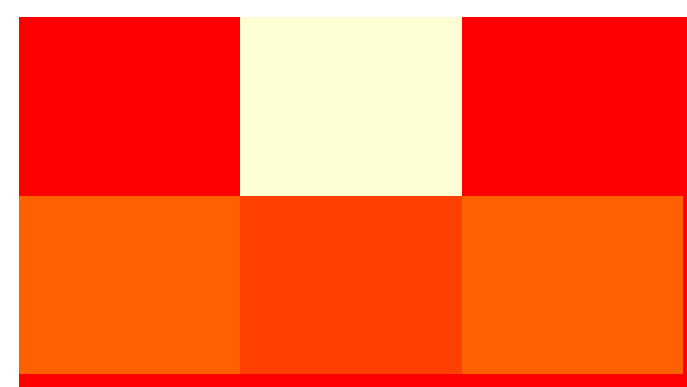

\begin{tabular}{|c|c|c|}
\hline 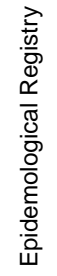 & 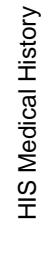 & 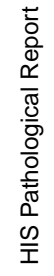 \\
\hline
\end{tabular}


QM Prostate Data Set

QM Cancer Diagnosis Data

HIS Surgery Urology

HIS Surgery Complications

HIS Pathological Report

HIS Medical History

Epidemological Registry

\begin{tabular}{|c|c|c|}
\hline 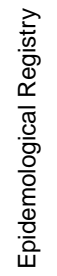 & 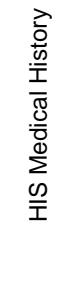 & 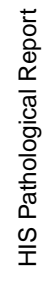 \\
\hline
\end{tabular}


Cluster Dendrogram of Forms

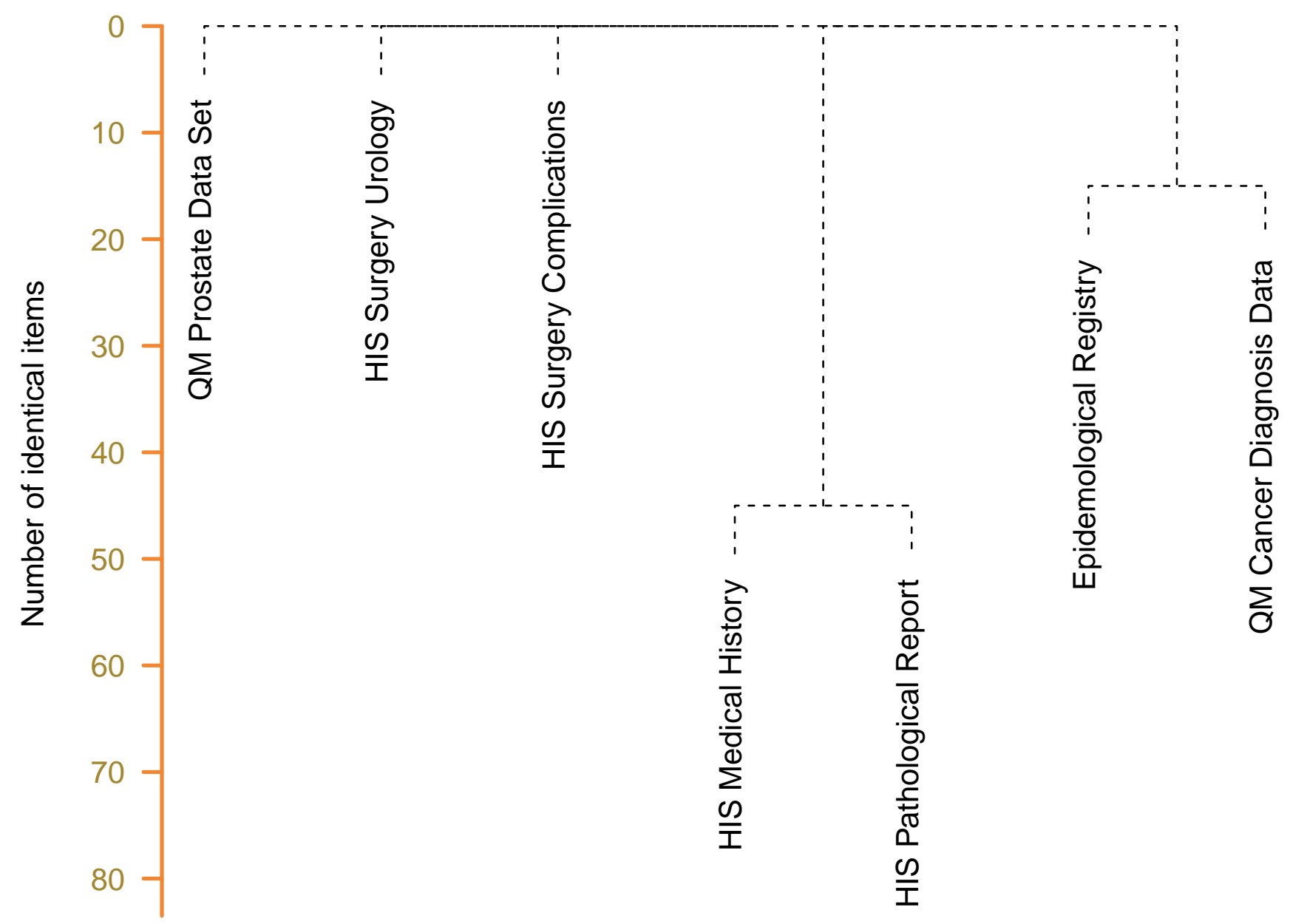

Form-Names 
Cluster Dendrogram of Forms

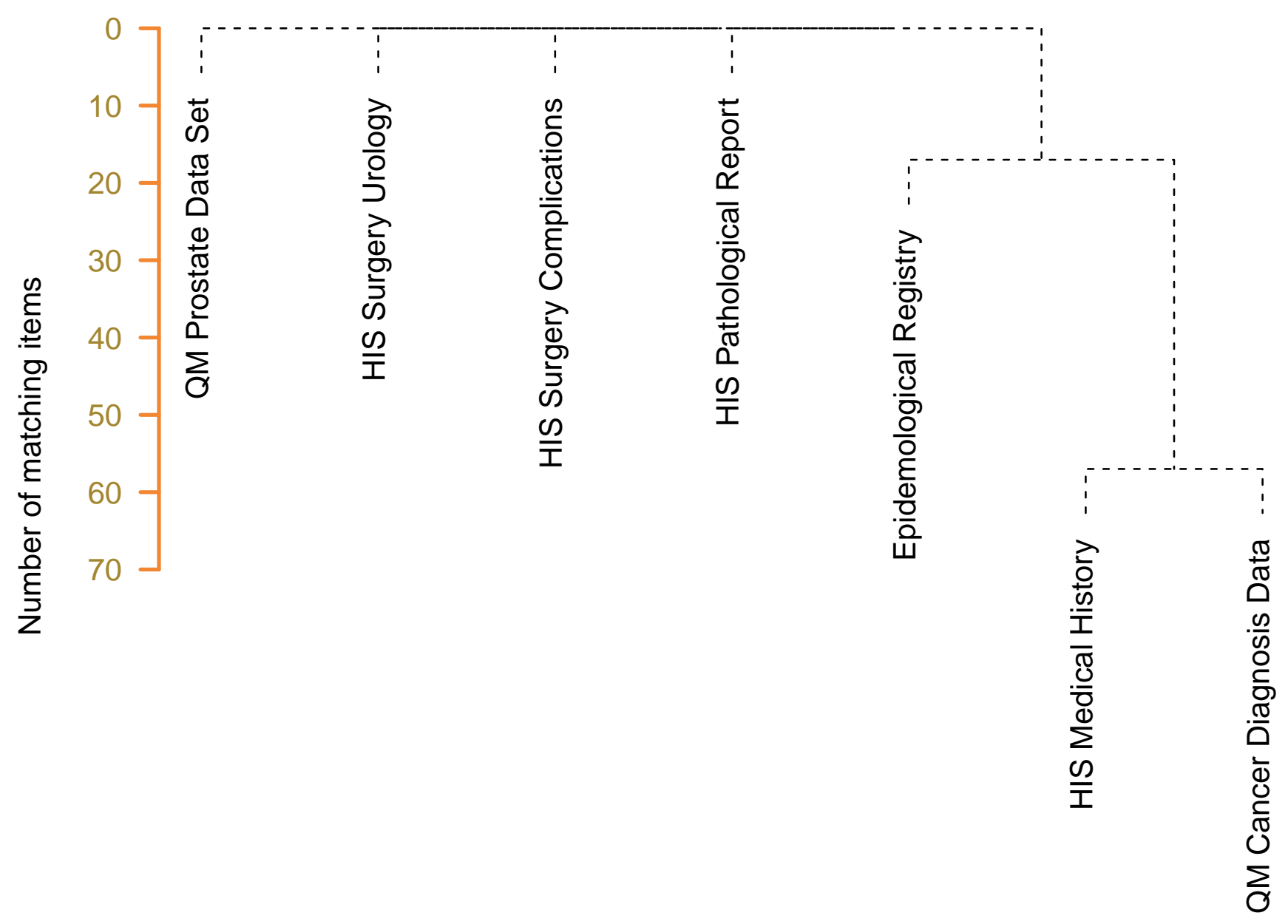

Form-Names 


\section{Cluster Dendrogram of Forms}

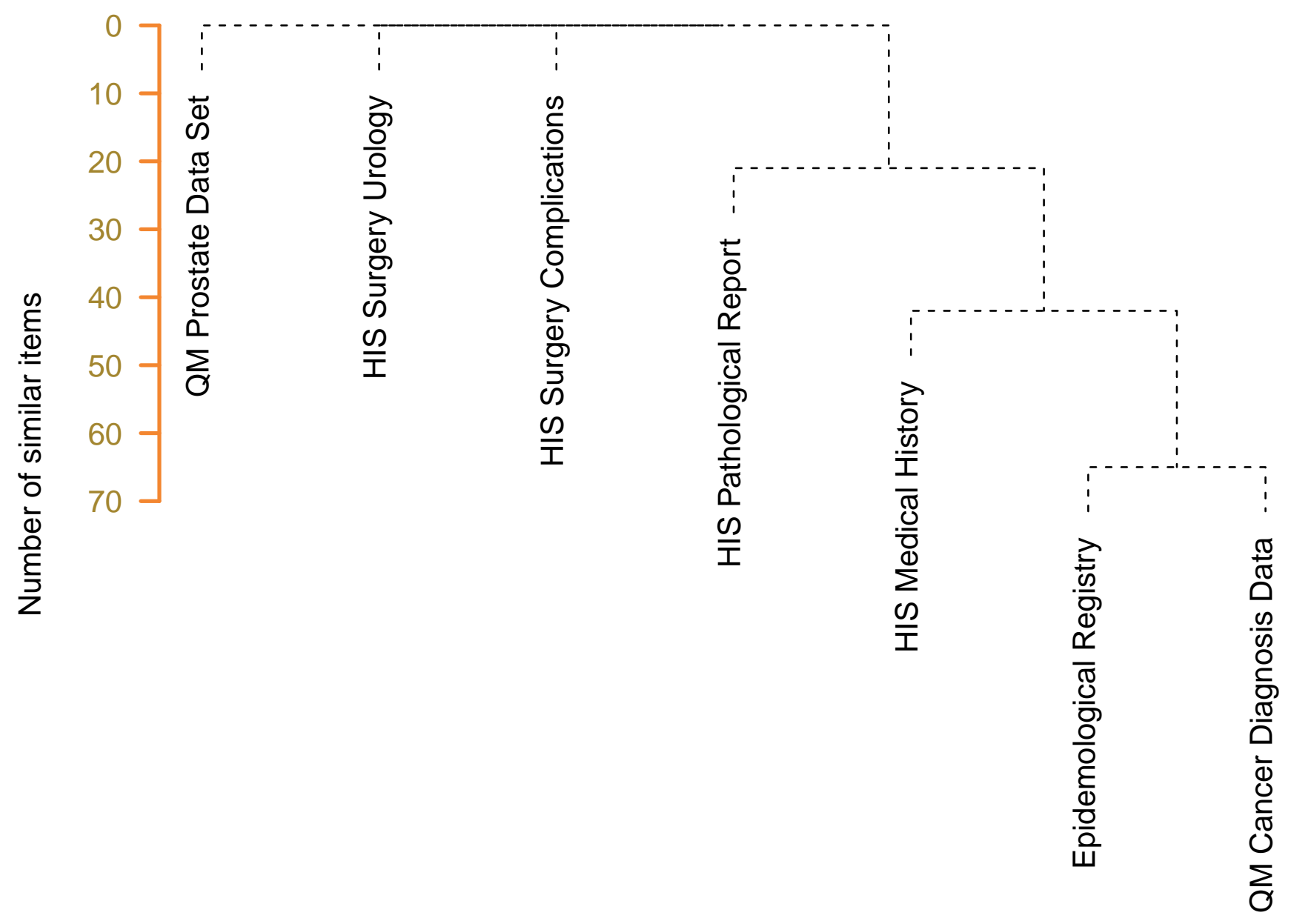

Form-Names 